\title{
Kernos
}

Revue internationale et pluridisciplinaire de religion grecque antique

7| 1994

Varia

\section{Un oracle relatif à l'introduction du culte de Cybèle à Athènes}

Angel Ruiz Pérez

\section{OpenEdition}

Journals

Édition électronique

URL : http://journals.openedition.org/kernos/1106

DOI : 10.4000/kernos. 1106

ISSN : 2034-7871

Éditeur

Centre international d'étude de la religion grecque antique

Édition imprimée

Date de publication : 1 janvier 1994

ISSN : 0776-3824

Référence électronique

Angel Ruiz Pérez, « Un oracle relatif à l'introduction du culte de Cybèle à Athènes », Kernos [En ligne], 7 | 1994, mis en ligne le 20 avril 2011, consulté le 03 mai 2019. URL : http://journals.openedition.org/ kernos/1106 ; DOI : 10.4000/kernos.1106 
Kernos, 7 (1994), p. 169-177.

\section{UN ORACLE RELATIF À L'INTRODUCTION}

\section{DU CULTE DE CYBÈLE À ATHÈNES}

Dans un colloque comme celui-ci, il n'est pas hors de propos de porter l'attention sur le sanctuaire oraculaire grec par excellence, Delphes, pour analyser son intervention dans le processus qui a transformé la religion hellénique au fil du temps. Notre but est d'observer si son influence visait à renforcer le pouvoir du culte des divinités grecques traditionnelles ou à introduire de nouveaux cultes étrangers, en comprenant sous ce terme les cultes qui n'avaient pas d'implantation en Grèce avant l'époque historiquel.

Une première démarche est d'analyser le corpus des réponses oraculaires que la tradition nous a transmises. Le premier résultat d'ensemble est surprenant : le sanctuaire mantique ne favorise pas le culte des divinités étrangères, sauf dans un cas remarquable, celui du culte de Cybèle. Nous nous limiterons à étudier celui-ci, afin de montrer les raisons de sa singularité.

Delphes a encouragé plusieurs fois le culte de divinités olympiennes ayant de nouvelles épithètes, et, dans quelques cas, l'introduction d'une nouvelle divinité a été facilitée par son assimilation avec la divinité la plus proche appartenant au groupe des Olympiens. Dans un seul cas, celui du roi Philippe II, l'oracle recommanda de vénérer une divinité non grecque, en l'occurrence égyptienne, $\mathrm{Ammon}^{2}$, mais uniquement parce que celui-ci était connu en Grèce en tant que Zeus Ammon. On trouve aussi un groupe d'oracles tardifs, qu'on pourrait qualifier de chrétiens $^{3}$, les seuls où l'on peut observer une confrontation de la Pythie avec un culte étranger, au point qu'elle déclare avoir été battue par le Christ. Mais il s'agit d'oracles tardifs, évidemment faux et créés par la propagande des groupes chrétiens.

1 Je tiens ici à remercier Alain Blomart, qui a eu l'amabilité de corriger le texte français et de me communiquer ses remarques.

2 H.W. PARKe \& D.E. W. WORMELL, The Delphic Oracle, Oxford, $1956^{2}$ (abrégé PW), n²69; J. FonTenrose, The Delphic Oracle, Berkeley, 1978 (abrégé F), $\mathrm{n}^{\circ} \mathrm{Q} 211$.

3 PW 518, 476/ F Q 250, 263, 268. 
C'est pour cela que le cas de l'oracle relatif à l'introduction du culte de Cybèle ${ }^{4}$ est particulier dans ce contexte, précisément parce que l'identification de la Grande Mère avec une autre déesse grecque ne s'est pas produite complètement. L'origine orientale de la Mère des dieux est connue, ainsi que son importance en Anatolie, pendant le deuxième millénaire aussi ${ }^{5}$, et la diffusion de son culte jusqu'en Grèce ${ }^{6}$, en passant par la Phrygie, où il a acquis une importance particulière à la fin du deuxième millénaire. Les Grecs d'Asie Mineure l'auraient adoptée au VIIe ou au VIe siècle av. J.-C. Son culte se répand surtout à partir de l'Ionie du Nord et de Cyzique ${ }^{7}$. Déjà Pindare ${ }^{8}$ la mentionne, à côté de Pan, en précisant aussi ses particularités cultuelles, et en l'appelant Cybèle, Mère des dieux ${ }^{9}$. Cette divinité recevait donc un culte déjà avant les guerres médiques, et ce par l'intermédiaire des Ioniens. Ses affinités, principalement avec Déméter, mais aussi avec Rhéa et Artémis, sont très bien connues. Cependant Cybèle conserve une personnalité caractéristique, même si les traits les plus barbares de son culte ont été adoucis, afin de rendre plus facile son entrée dans la sphère de la religion de l'État. Will considère comme élément décisif pour l'introduction de ce culte le fait que la Grande Mère soit arrivée sur le continent par l'Asie mineure, qui aurait servi de "filtre», surtout en séparant la déesse d'Attis, son amant, et en adoucissant plusieurs des traits «barbares» de son culte. La Grèce continentale aurait donc écarté ces aspects phrygiens de la déesse, tout en la reconnaissant encore comme déesse étrangère, et aurait préféré d'autres associations avec des

PW 572/F Q 133.

5 Cf. E. LAROCHE, Koubaba, déesse anatolienne, et le problème des origines de Cybèle, in Eléments orientaux dans la religion grecque ancienne. Colloque de Strasbourg, Paris, 1960, p. 113-28. Pour un bon résumé, à jour, voir W. BuRKerT, Structure and History in Greek Mythology and Ritual, Berkeley, 1979, p. 102-5.

Cf. A. HenRICHS, Despoina Kybele. Ein Beitrag zur religiösen Namenkunde, in $H S P h, 80$ (1976), p. 253-286. Le premier témoignage de la présence de Cybèle dans le monde grec a été étudié par M. GuarduCCI, Cibele in un'epigrafe arcaica di Locri Epizefiri, in Klio, 52 (1970), p. 133-8 et par J. DE LA GENIÈre, De la Phrygie à Locres Epizéfirienne, les chemins de Cybèle, in MEFRA, 97 (1985), p. 693-718.

7 Cf. F. Graf, Nordionische Kulte, Rome, 1985, p. 108 sq.

$8 \quad P y$. V, 77 sq., schol. V, 137 Drachmann, et fr. 79, 80, 95 Bergk.

9 Sur le nom de Cybèle et l'épiclèse Mère cf. C. BRIXHE, Le nom de Cybèle. L'antiquité avait-elle raison?, in Sprache, 25, 1 (1979), p. 40-45. 
figures grecques, principalement Pan et les Nymphes ${ }^{10}$. Burkert souligne l'existence dans le domaine grec de témoignages de vénération d'une déesse Mère depuis l'époque mycénienne ${ }^{11}$, ce qui a rendu possible l'acceptation de la Mère des dieux phrygienne. L'introduction de Cybèle en Grèce a donc été facilitée par l'existence d'un culte antérieur présentant des traits similaires. Elle a conservé sa personnalité particulière, tout en perdant quelques aspects caractéristiques: son iconographie - le naiskos, le lion, etc. - est restée la même tout au long de l'histoire grecque, malgré ces rapprochements.

Nous nous trouvons donc en face du seul oracle où une divinité étrangère, tout en conservant son nom et ses caractéristiques principales, est l'objet d'attention du sanctuaire de Delphes, qui recommande son culte pour Athènes. Pour étudier ce sujet, nous possédons surtout quelques témoignages narratifs tardifs. L'histoire est la suivante : un Metragyrtès, prêtre itinérant du culte de Cybèle, arriva à Athènes et essaya d'introduire les femmes au culte de la déesse. Pour des raisons que nous ne connaissons pas - mais qui sont sans doute communes à tous les cas d'introduction d'un culte extatique ou considéré comme étranger par les citoyens -, cela provoqua la colère des Athéniens, qui le tuèrent en le jetant dans le barathron. Survint alors une calamité peste ou famine. L'oracle de Delphes, alors consulté, expliqua la cause des malheurs et ordonna l'instauration du culte de la déesse - et aussi, selon une des sources, du culte du Metragyrtès - dans le Metrôon, partie du Bouleuterion qui avait la fonction de dépôt des archives de l'État ${ }^{12}$.

10 E. WILL, Aspects du culte et de la légende de la Grande Mère dans le monde grec, in Éléments orientaux (op. cit.), p. 95-110. Cf. aussi M.P. NILssoN, Geschichte der griechischen Religion, I, München, $1967^{3}$, p. 725-727; $\mathrm{Ph}$. Borgeaud, L'écriture d'Attis : le récit dans l'histoire, in C. Calame (éd.), Métamorphoses du mythe en Grèce ancienne, Genève, 1988, p. 87-103; ID., Comment lui trouver un nom?, in Les Mères. Nouvelle revue de psychanalyse, 45 (1992), p. 173-193; R.R. Simms, Foreign Religious Cults in Athens in the Fifth and Fourth Century B.C. (thèse de l'Univ. of Virginia), Charlottesville, 1985.

11 W. BuRKert, Greek Religion, Oxford, 1985, p. 177-9. Offrandes à une Mère déesse dans un texte en linéaire B de Pylos [PY Fr 1202]. L.R. FARNELL, The Cults of the Greek States, Oxford, 1896-1909, III, 296 sq., avait déjà souligné l'importance des témoignages sur une Mère divine en Crète, avec des traits iconographiques très semblables à ceux de Cybèle.

12 APOST., 11, 34 [= Souda, s.v. Metragyrtes, = PHOTIOS, I, 422 Naber]; Souda, s.v. barathron [= Schol. A RISTOPH., Pl., 431]; JULIEN, Or. V, 159b; Schol. EsCHINE in or. 3, 187. 
Comme on peut l'observer, la narration est assez stéréotypée et ses éléments fondamentaux correspondent à la généralité des types narratifs sur l'introduction du culte d'une divinité dans un lieu déterminé. Nous pouvons ainsi les schématiser ${ }^{13}$ :

a. Le Metragyrtès initie les femmes au culte de Cybèle.

b. Les Athéniens le tuent.

c. Un fléau survient et un oracle ordonne d'apaiser la déesse.

d. On construit un bouleuterion, qui est consacré à Cybèle [et on érige une statue du Metragyrtès].

e. Ce lieu devient dépôt d'archives; le barathron est couvert.

Les textes montrent une organisation très typique, à la manière de quelques autres récits d'introduction de cultes par un oracle ${ }^{14}$, par exemple les quatre cas de l'oracle de Delphes que Parker mentionne ${ }^{15}$; on ne peut donc pas croire à l'authenticité de l'histoire, au moins telle qu'elle est transmise par la tradition littéraire, car elle semble plutôt servir d'«aition» au barathron et au Metrôon : ainsi Apostolos écrit que le Metrôon a été construit là où le Metragyrtès est mort : autrement la connexion n'est pas possible. Vermaseren a pensé à une connexion entre la fonction de la Mère des dieux en tant que déesse de l'agriculture, grâce à l'assimilation à Déméter, et par conséquent protectrice de l'ordre humain et légal ${ }^{16}$. Cette solution, originale, n'empêche pas de considérer que la liaison entre le culte réel et l'histoire s'est faite d'une manière forcée. Si nous voulons suivre les traces d'autres témoignages sur l'antiquité du culte de Cybèle à Athènes, nous devrons faire appel aux restes archéologiques, principalement ceux qui ont été découverts à l'Agora athénienne. Aussi bien le Bouleuterion que le Metrôon, bâtiments unis, peuvent être datés entre les VIe et Ve siècles, comme

13 Cf. R. GARLAND, Introducing New Gods. The Politics of Athenian Religion, London, 1992, p. 152-170.

14 FARNELL, op. cit., III, p. 302, avait déjá écrit que the legend that the Athenians murdered the first metragyrtes who ventured to show himself in Athens is discredited.

15 H. Parker, Miasma. Pollution and Purification in early Greek Religion, Oxford, 1983, p. 272 sq. : ce sont les oracles PW 28, 331, 493, et 554.

16 M.J. Vermaseren, Cybele and Attis. The Cult and the Myth, London, 1977, p. 33-34. 
Vermaseren ${ }^{17}$ l'a démontré. Cependant, depuis Picard, une autre ligne de recherche a considéré que les restes antérieurs à 430 av. J.-C. n'ont jamais été un lieu du culte de Cybèle ${ }^{18}$. À l'appui de cette théorie, il y aurait la date de 430 , qui pourrait convenir au fléau décrit par les sources littéraires; la grande statue de la déesse réalisée en 430 par Phidias ou Agoracrite serait un autre indice que le culte a été introduit à Athènes à cette époque. À Athènes aussi, sur l'Acropole, on a découvert des représentations figurées de Cybèle, mais elles ne sont pas antérieures au IVe siècle av. J.-C. ${ }^{19}$. Au Pirée et à Agrai subsistent des témoignages d'un culte très répandu, mais également postérieurs au Ve siècle $^{20}$. Á Delphes, et ce point est important pour notre étude, il existe des représentations de Cybèle, très exactement trois, mais l'identification de Cybèle sur un relief du Trésor des Siphniens (datant de 530/25 av. J.-C.) a été rejetée récemment par Brinkmann ${ }^{21}$, et les autres restes ne sont pas aussi anciens.

17 M. J. Vermaseren, Corpus Cultus Cybelis Attidisque (CCCA), II Graecia atque insulae, Leiden, 1982, p. 3-4, distingue les phases suivantes :

a. Au début du Ve siècle, construction d'un petit temple $\left(20 \mathrm{~m}^{2}\right)$, pour la Mère des Dieux, à droite de l'ancien Bouleuterion. En 480/79, il est détruit par les Perses et n'est pas reconstruit. La déesse est placée dans l'ancien Bouleuterion, qui a aussi la fonction de conservation des archives de l'État.

b. À la fin du Ve siècle, construction d'un nouveau Bouleuterion et d'une salle de réunion. Une partie de l'ancien Bouleuterion contient les archives de l'État et la statue très connue sculptée par Phidias ou son disciple Agoracrite : ce bâtiment est appelé aussi Metrôon.

c. Dans le troisième quart du IIe siècle av. J.-C., on construit le Metrôon hellénis tique, avec quatre salles. La deuxième est le temple, et les autres ont la fonction de dépôt d'archives. L'ensemble a été détruit en 267 après J.-C.

Ch. PICARD, Die grosse Mutter von Creta bis Eleusis, in Eranos Jahrbuch, 6 (1938) [1939], p. 91-119.

VERMASEREN, CCCA, p. 17 et 49.

VERMASEREN, $C C C A$, p. 71 sq.

21 Vermaseren, CCCA, p. 136, n 441 et F. NEUMANn, Die Ikonographie der Kybele in der phrygischen und der griechischen Kunst (MDAI(I), Beiheft 28, 1983) avaient accepté l'identification avec Cybèle, mais V. BRINKMANN, Die aufgemalten Namenbeinschriften am Nord- und Ostfries des Siphnierschatzhauses, in BCH, 109 (1985), p. 77-130 - spécialement p. 101 - a démontré que la déesse qui est représentée sur la frise nord, montée sur un char tiré par deux lions et combattant un géant, était Thémis. Cf. aussi P. AMANDRY, $A$ propos de monuments de Delphes : Questions de chronologie (I), in $B C H, 112$ (1988), p. 591-610, spécialement p. 603 et 607. 
Ainsi, le culte de Cybèle, la Mère des dieux, existait à Athènes déjà au Ve siècle, et on a accepté jusqu'à nos jours qu'il y ait eu une représentation antérieure de la déesse à Delphes. C'est pour cela que les chercheurs ont accepté en général la vérité du récit sur l'oracle, en le datant de la même époque que les restes archéologiques les plus anciens, au commencement du Ve siècle, ou, dans le cas des adeptes de l'hypothèse de Picard, un peu plus tard, autour de 430 av. J.-C., pendant la Guerre du Péloponnèse. Parke ${ }^{22}$ appartient à ce dernier groupe et considère la date du dernier quart du Ve siècle comme la plus probable, à cause de l'importance de la statue sculptée par Phidias ou Agoracrite, son disciple, et surtout à cause d'un autre argument intéressant : dans la narration de l'oracle de Cybèle, on raconte que les Athéniens avaient abandonné le barathron en tant que place d'exécution, à cause de l'impiété qu'ils avaient commise, et la dernière date connue sur l'usage du barathron est 430 av. J.-C. ${ }^{23}$, une date, donc, qui s'accorde avec son hypothèse. Fontenrose ${ }^{24}$, pour sa part, accepte que la Mère des dieux asiatique soit arrivée à Athènes probablement à la fin du VIe siècle, lors de la première apparition des Metragyrtai. Pourtant il souligne le fait de l'existence de plusieurs anachronismes, par ailleurs explicables, dans l'ensemble des témoignages littéraires : par exemple, Julien, une des sources, parle de la Mère des dieux comme un gallos, terme inadéquat pour l'époque du récit. La Souda, pour sa part, raconte que le Metragyrtès a prédit l'arrivée de la Mère à la recherche de Corè : cela situerait le récit avant l'arrivée de Déméter à Éleusis; ce sont des anachronismes normaux, mais ils soulignent le caractère tardif de la création du récit. Fontenrose remarque que tous les témoignages sont aussi très tardifs : le premier, celui de Julien, appartient au IVe siècle après J.-C. En outre, seul Julien met en rapport cette histoire avec l'oracle de Delphes: les autres mentionnent un oracle, sans plus de précision.

La plupart des chercheurs, en s'appuyant sur les données archéologiques, datent donc la narration relative à l'oracle de Cybèle du début du Ve siècle ou de 430 , pour d'autres, dans le dernier cas à cause de l'identification du fléau avec la Guerre du Péloponnèse.

À notre avis, il serait préférable d'établir une différence nette entre la datation réelle du récit, c'est-à-dire la date de sa composition, et la

22 PW, I, p. 324-5.

23 La dernière reférénce au Barathron est THUC., II, 67, 4.

24 Commentaires à PW 572/Q 133, ad loc. 
date évoquée par son auteur. Ce qui plaide en faveur de cette différenciation, c'est principalement le caractère artificiel du récit, les anachronismes et sa narration typique : la création d'un espace de culte visant à apaiser une divinité irritée par une faute, ce qui provoque l'intervention oraculaire. À cela s'ajoute le caractère tardif des sources. En outre, il y a des raisons précises pour rejeter les dates anciennes de la légende, comme nous allons essayer de l'expliquer à présent.

En contradiction directe avec la date de 430 se trouve, à notre avis, l'introduction officielle à Athènes, la même année, du culte de Bendis, la déesse thrace; elle est attestée dans un passage célèbre de $L a$ République ${ }^{25}$. Platon y raconte que Socrate est allé, le $19 \mathrm{du}$ mois de Thargéliôn ${ }^{26}$, à la fête de Bendis, célébrée pour la première fois. L'instauration de ce culte à Athènes est un fait qui obéit, selon l'opinion commune, à une politique diplomatique entre Athènes et la Thrace, précisément au début de la Guerre du Péloponnèse ${ }^{27}$. Dans ce cas, Delphes n'a pas été consultée comme c'était l'habitude en l'espèce; par Thucydide ${ }^{28}$ nous savons que la position de Delphes au début de la guerre était clairement favorable à Sparte, et on possède des données qui certifient qu'Athènes a consulté Dodone au lieu de Delphes pour l'introduction du culte de Bendis; dans une inscription postérieure, on lit que seule Bendis a été acceptée à Athènes par l'intermédiaire d'un oracle ${ }^{29}$. Accepter cette date comme celle de l'introduction du culte de Cybèle devient extrêmement difficile, vu qu'à la même époque un culte étranger était accepté par l'intermédiaire de Dodone et non de Delphes : par l'archéologie, l'existence d'un culte de Cybèle à Athènes est attestée, mais on ne peut pas en dire autant pour ce qui est de l'information litté-

$25 \quad 327$ a et $328 \mathrm{a}$.

26 Cf. Proclus, In Tim., 17b.

27 Cf. M.P. NILSSON, op. cit., p. 883-884 et H.W. PARKE, Festivals of the Athenians, London, 1977, p. 149-152. Cf. aussi les travaux récents de R. GARLAND, op. cit, spéc. p. 111-115; ID., The Piraeus: from the Fifth to the First Century B.C., London, 1987, spéc. p. 118-122 et 231-33; R.R. Simms, The Cult of the Thracian Goddess Bendis in Athens and Attica, in AW, 18 (1988), p. 59-76; A. B LOMART, Bendis à Athènes. L'intégration d'une déesse étrangère, D.E.A. non publié, Paris, École Pratique des Hautes Etudes (Sciences Religieuses), 1992.

I, 118, 3; cf. R.R. SIMMS, The Cult..., art. cit., p. 62.

29 Cf. L. Ziehen-J. von Prott, Leges Graecorum Sacrae, Leipzig, 1896-1906, $\mathrm{n}^{\circ} 42$, du 263/2 av. J.-C., spéc. 1. 4-6 ( = IG $\mathrm{II}^{2}, 1283$ ) et le commentaire ad loc. Cf. aussi PARKE, Festivals, op. cit., p. 150. 
raire transmise sur l'oracle d'introduction du culte. Une date plus ancienne pour cette narration est de même assez improbable, pour des raisons intrinsèques au texte en question : son caractère artificiel nous semble un signe assez clair de composition tardive, et on peut en dire de même, dans une certaine mesure, des anachronismes.

À notre avis, une date plus tardive pour la narration de l'oracle serait davantage fondée. Les raisons internes - structure typique de l'histoire, sources trop tardives, anachronismes évidents - et externes introduction parallèle du culte de Bendis par l'intermédiaire de Dodone - peuvent nous faire comprendre plus clairement que nous nous trouvons ici devant une narration tardive, qui veut se faire passer pour archaïque. Une date possible, à notre avis, est le IIIe siècle, où le culte de Cybèle, avec ses traits les plus caractéristiques, est bien implanté en Grèce, et à la fin du même siècle aussi à Rome, par l'intermédiaire d'un oracle bien documenté de Delphes. À cet égard, le rôle de la dynastie de Pergame, les Attalides fut importante, parce que nous connaissons ses relations d'amitié aussi bien avec Delphes ${ }^{30}$ qu'avec Athènes ${ }^{31}$, et son rôle essentiel dans l'introduction du culte de Cybèle à Rome ${ }^{32}$, en 204 av. J.-C. D'autre part, la Mère des dieux avait déjà un culte à Pergame à l'époque de Philétère, le fondateur de la dynastie attalide ${ }^{33}$, et on

30 De forts liens avec Delphes apparaissent dès l'époque d'Attale I : il a bâti une terrasse à côté du temple d'Apollon : cf., pour les monuments des Attalides à Delphes, J.-F. Bommelaer, Guide de Delphes. Le site, Paris, 1991, p. 191-195, et, pour les inscriptions, Fouilles de Delphes, III, 3, 240 et 261; dans cette dernière inscription on lit expressément - 1, 2-5 - qu'on honore spécialement aussi bien Eumène II qu'Attale II parce qu'ils ont toujours été les promoteurs des relations entre Delphes et Rome, le nouveau pouvoir de la Grèce. Cf. aussi R.E. AlLEN, The Attalid Kingdom. A Constitutional History, Oxford, 1983.

31 À Athènes, un sacerdoce d'Attale I a été créé en 210 av. J.-C. : cf. IG II $^{2} 5080$, et une tribu a reçu son nom en 200 av. J.-C. : cf. PoL., XVI, 25, 9.

32 Cf. M.J. Vermaseren The Cult..., op. cit. p. 38-41; G. Thomas, Magna Mater and Attis, in ANRW, II, 17.3 (1984), p. 1500-1535; D.M. CosI, L'ingresso di Cibele ad Atene e Roma, in CDRAC, 1980-1981, p. 81-91.

33 Cf. E.V. HANSEn, The Attalids of Pergamon, Ithaca (N. Y.), 1947, p. 398-399; E. OHLemutz, Die Kulte und Heiligtümer der Götter in Pergamon, Würzburg, 1940. Cf. aussi F. GRAF, op. cit., p. 114-115. On a découvert à Pergame des inscriptions à la Meter Basileia : IvP 334, 481-3, d'époque romaine, et aussi une statuette de Cybèle du IIe siècle av. J.-C., avec une inscription sur la base (Cf. CIG $68_{35}$; V. MÜLLER, Eine Statuette der Kybele in Wien, in $R h M, 34$ (1919), p. 82-106). Deux temples de Pergame appartenaient à la Mère des dieux : celui de Mamurt-Kaleh, érigé par Philétère (cf. A. CONZE, 
connaît les relations amicales entre certains Attalides et les prêtres du temple de la Mère à Pessinonte ${ }^{34}$. Il est donc possible qu'au cours de cette période ait été créée la légende selon laquelle le sanctuaire pythique s'attribuait l'honneur d'avoir été l'introducteur du culte d'une déesse de plus en plus importante dans l'œe umenè, et principalement dans l'empire en émergence, Rome, et avant aussi à Athènes, ce qui donnerait à Delphes, en tant que promoteur de ce culte en Grèce, l'autorité suffisante pour le recommander à l'empire qui commençait à dominer le monde. La collaboration de Delphes, dont le prestige était grand, avec la dynastie attalide, dominante dans la zone où le culte de la déesse était plus important - le mont Ida, Pessinonte - était par conséquent hautement profitable pour les deux parties. Une histoire typique, facile à réaliser, a servi pour ces buts. En plus, nous possédons un oracle de cette période ${ }^{35}$ ( 250 av. J.-C.), qui conseille à Posidonios d'Halicarnasse de vénérer divers dieux et, parmi eux, la Mère des dieux.

Nous pouvons conclure en disant que Delphes ne semble pas avoir été un lieu de propagation des cultes non grecs, et que c'est seulement à partir du IIIe siècle, par la force des événements extérieurs - l'influence de la dynastie attalide, dominante dans l'Orient grec, l'expansion de facto du culte de Cybèle à cette époque -, elle s'attribuera une fonction centrale dans l'apparition d'un culte étranger, en le reconnaisant comme tel. Avant cette époque, c'est le traditionalisme en matière religieuse qui semble avoir caractérisé l'oracle delphique.

Departamento de Filología Griega

Angel RuIz PÉrez

Facultad de Filosofía y Letras

Plaza de la Universidad, 2

E -47003 VALLADOLID

P. Schatzmann, Mamurt-Kaleh. Ein Tempel der Göttermutter unweit Pergamon (JDAI Erg. h. 9), Berlin, 1911) et le Megalesium. Il est très probable qu'il existait un autre temple sur l'Acropole de Pergame (Cf. HANSEN, op. cit., p. 399).

34 Cf. W. DitTenBerger, Orientis Graeci Inscriptiones Selectae, Leipzig, 19031905, n. 315 : des lettres d'Eumène II et Attale II pour Attis, le prêtre de la Mère à Pessinonte : on peut remarquer qu'il est dit que les Attalides ont toujours protégé le sanctuaire.

FH 36. 